\title{
Central corneal thickness in a Jordanian population and its association with different types of Glaucoma: cross-sectional study
}

\author{
Sana' Muhsen ${ }^{1}$, Feras Alkhalaileh², Mohammad Hamdan² and Saif Aldeen AlRyalat ${ }^{3 *}$ (D)
}

\begin{abstract}
Background: Central corneal thickness (CCT) has long been implicated to affect glaucoma predisposition. Several reports have identified that thinner CCT is a risk factor for open-angle glaucoma, and that CCT can be very variable between different ethnic groups. In this study, we aim to identify the relation between CCT and different glaucoma parameters in different types of glaucoma in an Arabian ethnicity.

Methods: We classified our participants into four main groups: primary open-angle glaucoma (POAG), primary angleclosure glaucoma (PACG), pseudoexfoliative glaucoma (PXFG), and a control group. We obtained demographics, intraocular pressure (IOP), cup to disc ratio (CDR), visual field mean deviation (MD) and pattern standard deviation (PSD), CCT, and retinal nerve fiber layer (RNFL) thickness for each participant.

Results: We included A total of 119 eyes with glaucoma, including POAG (54 eyes), PXFG (31 eyes) and PACG (34 eyes), we also included 57 control eyes. We found that PACG eyes have the thinnest CCT. Mean measurements of CCT for our groups were: $538.31 \mu \mathrm{m}(\mathrm{SD}=36.30)$ in eyes with POAG, $544.45 \mu \mathrm{m}(\mathrm{SD}=28.57)$ in eyes with PXFG, $506.91 \mu \mathrm{m}$ (SD $=34.55)$ in eyes with PACG and $549.63 \mu \mathrm{m}(\mathrm{SD}=42.9)$ in the control group. We found that $C C T$ is significantly correlated with CDR $(p=0.012, r=-0.231)$, MD $(p<0.001, r=0.327)$, and RNFL thickness $(p=.007, r=.283)$.

Conclusion: In Arabian ethnicity, PACG patients have the thinnest CCT compared to other types of glaucoma, namely POAG and PXFG. We demonstrated that glaucomatous eyes with thinner corneas will probably have more advanced glaucomatous optic neuropathy. Our results emphasize the importance of taking ethnicity into account upon glaucoma management.
\end{abstract}

Keywords: Glaucoma, Central corneal thickness, Ethnicity

\section{Background}

Glaucoma is the second leading cause of blindness in the world, after cataracts, and is the leading cause of blindness among African-Americans [1]. It is generally classified into open-angle and closed-angle glaucoma, and both can be either primary or secondary. It is estimated that the number of people with primary open-angle glaucoma (POAG) worldwide will reach 58.6 million by 2020 , while 21 million will be affected by primary angle-closure glaucoma (PACG)

\footnotetext{
* Correspondence: saifryalat@yahoo.com

${ }^{3}$ Department of Ophthalmology, University of Jordan Hospital, The University of Jordan, Amman 11942, Jordan

Full list of author information is available at the end of the article
}

[1]. Of those, 5.9 and 5.3 million will be blind from irreversible optic nerve damage associated with POAG and PACG, respectively [1]. This means that early detection and identification of risk factors are key elements in controlling the disease and preventing its progression. Secondary open-angle glaucoma is another entity with diverse types, but Pseudoexfoliation glaucoma (PXFG) is currently the leading cause of secondary open-angle glaucoma with a prevalence reaching $40 \%$ in patients over the age of 80 [2], and is highly dependent on race and ethnicity [3].

Evidence in the recent literature has shown the importance of central corneal thickness (CCT) in relation to several ocular conditions. Most notably, thinner CCT

(c) The Author(s). 2018 Open Access This article is distributed under the terms of the Creative Commons Attribution 4.0 International License (http://creativecommons.org/licenses/by/4.0/), which permits unrestricted use, distribution, and reproduction in any medium, provided you give appropriate credit to the original author(s) and the source, provide a link to the Creative Commons license, and indicate if changes were made. The Creative Commons Public Domain Dedication waiver (http://creativecommons.org/publicdomain/zero/1.0/) applies to the data made available in this article, unless otherwise stated. 
has been identified as a risk factor for open-angle glaucoma [4]. Goldmann and Schmidt discussed the association between the corneal thickness and the intra-ocular pressure (IOP) in their publication in 1957 [5]. Fourteen years later, Hansen and Ehlers demonstrated the presence of a positive linear correlation between CCT and IOP [6]. More recently, a meta-analysis of worldwide CCT literature proposed a correction factor of $2.5 \mathrm{mmHg}$ for each $50 \mu \mathrm{m}$ change in CCT [7].

CCT can be very variable between different ethnic groups, as shown in studies comparing mean CCT in both normal and glaucomatous eyes between Caucasian, Hispanic, African American, and Asians, where the thinnest corneas being more prevalent among African American ethnicity [8, 9]. Other studies also showed significant variation in CCT between ethnic sub-groups, as in a study that included different Asian populations; Chinese, Japanese, Korean, Filipino, Pacific Islander, and South Asian, and showed that the thinnest corneas are found in South Asian populations [10]. Moreover, several studies have shown that CCT varies among individuals with different types of glaucoma (POAG, PACG, and PXFG) and normal eyes, as in Bechmann et al. study that showed thinner corneas in patients with PXFG and POAG compared with patients with PACG and normal eyes [11]. In Jordanian population; an Arabian, Middleeastern ethnicity, previous studies showed that glaucoma is one of the leading causes of blindness [12], and at the same time, the level of knowledge about glaucoma among Jordanians is poor (around 75\% have either no or low level of knowledge) compared to other parts of the world [13], leading to delayed diagnosis of this silent disease. Ophthalmologists in Jordan usually depend on studies that were done on other ethnicities upon treating their glaucoma patients, due to lack of studies on the characteristics of glaucoma among Jordanians. Given the importance of early detection of risk factors of glaucoma, and as corneal thickness is one of the main risk factors for glaucoma and is the one associated with ethnicity, we aim to assess the corneal thickness in different types of glaucoma and in normal controls in a Jordanian population. We further aim to explore the relationship between CCT and the severity of glaucomatous optic neuropathy in different types of glaucoma.

\section{Methods}

\section{Participants}

This is a cross-sectional observational study that was conducted on subjects visiting the glaucoma clinics in 2 centers in Jordan between November 2015 and May 2017. We obtained ethical approval from the Institutional Review Board (IRB) and all patients were consented and approved to participate in this study.
This study was conducted in accordance with the Declaration of Helsinki latest update (2013). We included both patients with glaucoma and age-gender matching controls. Glaucoma patients were classified into three types of glaucoma: POAG, PACG, and PXFG. A diagnosis of POAG or PACG was made based on IOP, gonioscopy and both characteristic visual field (VF) defects (nasal step, arcuate scotoma, and paracentral defect), and optic nerve changes (enlarged cup to disc ratio (CDR), localized notch, and disc hemorrhage) in at least 1 eye as well as Ocular Coherence tomography (OCT) showing thinning of Retinal Nerve Fiber Layer (RNFL). For PXFG, pupils were dilated and exfoliation was checked and recorded as present or absent. PXFG was defined as an open-angle glaucoma with concomitant exfoliation material observed at pupillary border or anterior lens capsule with dilated pupil that is associated with glaucomatous optic neuropathy, as defined earlier.

To be included in this study the patients had to have all of the following inclusion criteria:

- Adult patients (age more than 18 years).

- A diagnosis of POAG, PACG or PXFG in at least 1 eye.

- A reliable VF examination taken within 3 months of the pachymetry. Reading had to be available for both eyes.

If the subject had any of the following exclusion criteria he/she was exempted from the study:

- Glaucoma types other than the aforementioned three types.

- Corneal pathology or surgery that might influence pachymetry.

- Underwent a cataract surgery.

- Patients with systemic diseases that might result in VF changes.

- Keratoconus.

- Contact lens use.

- Corneal dystrophy.

For controls, we included them from general ophthalmology clinics with matching gender and age ( \pm 2 years), and with a corneal topography of acceptable quality. We adopted the following exclusion criteria:

- Being a first or a second degree relative of any of our included patients.

- Keratoconus or other corneal disease

- History of Glaucoma or clinical signs of glaucoma

- History of corneal or intraocular surgery or trauma

We ran a power analysis to calculate the minimum sample size required for our study, the sample size was 
calculated based on the following assumptions: Effect size based on previous studies (see discussion) $=0.59$; power $=80 \%$; and two-sided alpha level $=0.05$. The required total sample size was 90 .

\section{Parameters measured}

Diagnosis of glaucoma and classification was confirmed by the attending ophthalmologist based on IOP measurements, gonioscopy, optic nerve changes, visual field defects and OCT RNFL thinning as stated before. We also asked included patients about their demographic data including age, gender, and ethnicity, as well as their previous ocular and medical history.

We measured the IOP using a Goldmann applanation tonometer, where our consultant ophthalmologist performed three measurements for each participant, and the average value was calculated. IOP readings included in the analysis were of patients treated with one or more antiglaucoma eye drops. Gonioscopy was done using a 4-mirror Sussman lens to classify angles into open or closed and to illicit signs of PXFG. A detailed anterior segment examination by slit lamp was performed to rule out corneal pathology that would exclude the patients from the study and also to help detect pseudoexfoliation. Optic nerve assessment including cup-to-disc ratio (CDR) using indirect biomicroscopy and a super field lens were performed by the consultant ophthalmologist.

We measured CCT via Oculus Pentacam HR for both glaucoma patients and controls. The Pentacam HR is a high-resolution rotating Scheimpflug camera system for anterior segment analysis. It provides crisp images of cornea, iris and lens. An ophthalmic technician performed the imaging during daytime (from 9:00 am to 4:00 pm). We then analyzed the Pentacam printouts to rule out any corneal pathologies, such as keratoconus, that would exclude patients from our study and to determine the central corneal thickness at the apex.

Other parameters were assessed for glaucoma patients only. Visual Field assessment was performed using Oculus Centerfield analyzer with a screening 24-2 Threshold strategy. RNFL thickness measurement was performed using Optovue RTVue Optical Coherence Tomography OCT, which generates high-resolution, cross-sectional (3D) images of the retina, optic disc and anterior segment.

\section{Statistical analysis}

We used SPSS 21.0 (Chicago, USA) in our statistical analysis. We first described our sample population and eyes included via descriptive statistics including numbers (percentages) and mean $(+\backslash$ - standard deviation SD).

We used logistic regression with generalized estimating equation to analyze effect of gender on type of glaucoma developed and eyes included. We used Independent sample t-test to study differences in general parameters and both the gender and the eye involved. To account for the within-subject effect, we used one-way repeated measure univariate analysis to study the relation between type of glaucoma and general parameters, followed by post-hoc analysis using Tukey analysis. We used Pearson's correlation to study the correlation between age and the other parameters. We also performed Spearman's test, after controlling for gender, to confirm association results. We finally adopted a model building strategy for a regression analysis to find predictors of visual field mean deviation (MD), where we corrected for age and gender. We inspected our data visually using boxplots and histograms, and we used Levene's test to check for homogeneity of variance. We also used Mauchly's test for sphericity to apply univariate analysis. We used a threshold of 0.05 for $p$ value to indicate statistical significance.

\section{Results}

We included a total of 68 Jordanian patients in this study, with a mean age of 65.9 years $(\mathrm{SD}=8.9)$. There were $42(62 \%)$ men and 26 (38\%) women. From the sample included, 119 eyes met our inclusion criteria and were included in our study, they were $61(51.3 \%)$ right eyes and 58 (48.7\%) left eyes (51 bilateral and 17 unilateral eyes). Eyes with glaucoma were categorized according to type of glaucoma into: POAG (54 eyes), PXFG (31 eyes) and PACG (34 eyes), as shown in (Table 1). We also included 29 control participants with a mean age of 55 years. They were 14 men and 15 women and a total of 57 eyes.

Upon analyzing the correlation between gender discrepancy and type of glaucoma developed, we found a significant difference $(p<0.001)$ as men were more likely to develop POAG (55.4\%) compared to $28.9 \%$ for women. However women were more likely to develop PACG (44.4\%) compared to only $18.9 \%$ for men. No significant difference was found between gender discrepancy and the development of PXFG. We found no statistically significant difference between both genders regarding other parameters. Mean values of different glaucoma-related parameters for both genders are detailed in (Table 2).

Regarding central corneal thickness (CCT), our results showed the following mean measurements: $538.31 \mu \mathrm{m}$ $(\mathrm{SD}=36.30)$ in eyes with POAG, $544.45 \mu \mathrm{m}(\mathrm{SD}=28.57)$ in eyes with PXFG, $506.91 \mu \mathrm{m}(\mathrm{SD}=34.55)$ in eyes with PACG, and $549.63 \mu \mathrm{m}(\mathrm{SD}=42.9)$ in the control group. First, we studied the difference between CCT of each glaucoma type and the control group, we found that subjects with PACG had significantly thinner corneas than the control group $(p=0.021)$. No statistically 
Table 1 General descriptive for the main three types of glaucoma and control group

\begin{tabular}{|c|c|c|c|c|}
\hline & \multicolumn{3}{|l|}{ Type of Glaucoma } & \multirow[t]{2}{*}{ Control } \\
\hline & Primary Open Angle Glaucoma & Closed Angle Glaucoma & Pseudoexfoliation Glaucoma & \\
\hline \multicolumn{5}{|l|}{ Gender } \\
\hline Male Frequency (\%) & $41(75.9 \%)$ & $14(41.2 \%)$ & 19 (61.3\%) & $27(47.4 \%)$ \\
\hline Female Frequency (\%) & $13(24.1 \%)$ & $20(58.8 \%)$ & $12(38.7 \%)$ & $30(52.6 \%)$ \\
\hline \multicolumn{5}{|l|}{ Age } \\
\hline Mean $( \pm \mathrm{SD})$ & $64.26( \pm 9.18)$ & $66.85( \pm 9.98)$ & $67.8( \pm 6.93)$ & $54.85( \pm 10.9)$ \\
\hline \multicolumn{5}{|l|}{ Eye } \\
\hline Right Frequency (\%) & $27(50 \%)$ & $18(52.9 \%)$ & $16(51.6 \%)$ & $28(49.1 \%)$ \\
\hline Left Frequency (\%) & $27(50 \%)$ & $16(47.1 \%)$ & 15 (48.4\%) & 29 (50.9\%) \\
\hline
\end{tabular}

significant difference was found between CCT of control group and any of POAG or PXFG groups $(p=.702)$. Upon comparing mean CCT between glaucoma groups, we found a significant difference between the groups in general $(p<0.001)$, so we performed a post-hoc analysis to find the group differences as shown in (Table 3).

Upon studying the relation between CCT and parameters of optic nerve damage (CDR, MD, and RNFL) in patients with glaucoma, we found that CCT is negatively and significantly correlated with CDR $(p=0.012, r=-$ 0.231 ), so that the thinner the cornea, the higher the CDR. We also found a significantly positive correlation between CCT and MD $(p<0.001, r=0.327)$, so that the thinner the cornea the lower the MD (more advanced visual field loss). Finally, we found a positive correlation between CCT and RNFL ( $p=.007, r=.283$ ) meaning that the thinner the cornea, the thinner the RNFL. No significant correlation was found between CCT and either IOP or pattern standard deviation (PSD).

We studied factors predicting visual field mean deviation (MD), and found that it is significantly associated with both CDR $(p<0.001)$ and RNFL $(p=0.003)$. Upon analyzing the relation between type of glaucoma and our measured parameters, we found several significant relations; our results are summarized in (Table 2). No statistical significance was found between either the age or laterality of the involved eye (left or right) on one hand and type of glaucoma or other measured parameters on the other hand.

\section{Discussion}

This study was done on a Jordanian population, a poorly studied population regarding glaucoma and its risk factors. The mean central corneal thicknesses (CCT) of normal adults and patients with 3 different types of glaucoma was measured by corneal topography. We also established that CCT in patients with PACG is significantly thinner than in other glaucoma types and in the control group. As visual field defect is an important outcome in glaucoma, we found that both cup to disc ratio (CDR) and retinal nerve fiber layer (RNFL) thickness, as measured by Optical Coherence Tomography, are the main factors associated with lower mean deviations on visual field testing. Gender discrepancy in glaucoma type preferences found to be in concordance with some previous reports where men are more likely to develop

Table 2 Gender differences of the mean glaucoma parameters measured in this study among glaucoma patients

\begin{tabular}{lllll}
\hline & Gender & Mean & Std. Deviation & Std. Error Mean \\
\hline Intra-ocular Pressure & Male & 15.1739 & 4.49347 & 0.66253 \\
& Female & 15.6724 & 5.12744 & 0.95214 \\
Cup to Disc Ratio & Male & 0.6234 & 0.27892 & 0.03242 \\
& Female & 0.5587 & 0.24368 & 0.03633 \\
Mean Deviation & Male & -5.5229 & 5.97786 & 0.71965 \\
& Female & -6.6430 & 5.83435 & 0.88973 \\
Pattern Standard Deviation & Male & 3.9250 & 1.72405 & 0.21222 \\
Retinal Nerve Fiber Layer Thickness & Female & 4.2298 & 1.51917 & 0.23167 \\
& Male & 77.5424 & 21.27373 & 2.47302 \\
Central Corneal Thickness (CCT) & Female & 78.3881 & 19.10243 & 3.91309 \\
& Male & 534.5270 & 34.14763 & 3.96958 \\
\hline
\end{tabular}


Table 3 Relation between type of glaucoma and different parameters

\begin{tabular}{|c|c|c|c|c|c|c|}
\hline Parameter & $\begin{array}{l}\text { Primary Open } \\
\text { Angle Glaucoma }\end{array}$ & $\begin{array}{l}\text { Closed Angle } \\
\text { Glaucoma }\end{array}$ & $\begin{array}{l}\text { Pseudoexfoliative } \\
\text { Glaucoma }\end{array}$ & $\begin{array}{l}\text { Between subject } \\
\text { effect } p \text {-value }\end{array}$ & $\begin{array}{l}\text { Mean difference } \\
(95 \% \mathrm{Cl})\end{array}$ & $\begin{array}{l}\text { Post-hoc } \\
p \text {-value }\end{array}$ \\
\hline $\begin{array}{l}\text { Intraocular pressure } \\
(\mathrm{mmHg})\end{array}$ & $15.2(4.3)$ & $13.6(3.2)^{*}$ & $17.3(5.8)^{*}$ & 0.049 & * $3.7(0.1-7.2)$ & ${ }^{*} 0.04$ \\
\hline Cup to disc ratio & $0.61(0.25)$ & $0.72(0.18)^{*}$ & $0.44(0.29)^{*}$ & 0.006 & * $0.3(0.8-0.47)$ & ${ }^{*} 0.004$ \\
\hline $\begin{array}{l}\text { Retinal nerve fiber } \\
\text { layer thickness }(\mu \mathrm{m})\end{array}$ & $80.5(20.8)$ & $70.8(18.2)$ & $82.2(20.9)$ & 0.084 & - & - \\
\hline $\begin{array}{l}\text { Central corneal } \\
\text { thickness }(\mu \mathrm{m})\end{array}$ & $538.3(36.3)^{*}$ & $506.9(34.6)^{* \#}$ & $544.5(28.6)^{\#}$ & $<0.001$ & $\begin{array}{l}\text { * } 46.2(21.9-70.6) \\
\text { \# } 36.6(10.5-62.7)\end{array}$ & $\begin{array}{l}*<0.001 \\
\# 0.004\end{array}$ \\
\hline $\begin{array}{l}\text { Visual field mean } \\
\text { deviation }(\mathrm{dB})\end{array}$ & $-4.9(5.6)^{*}$ & $-9.0(6.8)^{* \#}$ & $-4.7(4.4)^{\#}$ & $<0.001$ & $\begin{array}{l}7.3(3.5-11.1) \\
\# 7.5(3.5-11.6)\end{array}$ & $\begin{array}{l}*<0.001 \\
\#<0.001\end{array}$ \\
\hline Visual field Pattern standard deviation & $4.0(1.7)$ & $4.1(1.8)$ & $4.1(1.5)$ & 0.351 & - & - \\
\hline
\end{tabular}

Mean reading for each parameter and its standard deviation are presented. Significant differences between any two readings as analyzed by post-hoc analysis were marked by either $(*)$ or (\#)

POAG [14], and women are at higher risk for PACG, likely due to anatomical predisposition [15]. (Fig. 1) shows images for a patient with advanced chronic angle closure glaucoma and with significantly thin central corneal thickness, where the visual field test demonstrating a superior arcuate scotoma splitting fixation and ocular coherence tomography of the retinal nerve fiber layer thickness (OCT RNFL) shows significant thinning.
Previous studies, that were done on western populations, aimed to find the relation between CCT and other parameters, including visual field defects, have mainly included POAG eyes, and they found significantly more advanced visual field defects for lower CCT compared to thicker ones $[16,17]$. Here, we studied different types of glaucoma patients and control participants from a Jordanian population, and we found similar correlations where patients with lower CCT had significantly more

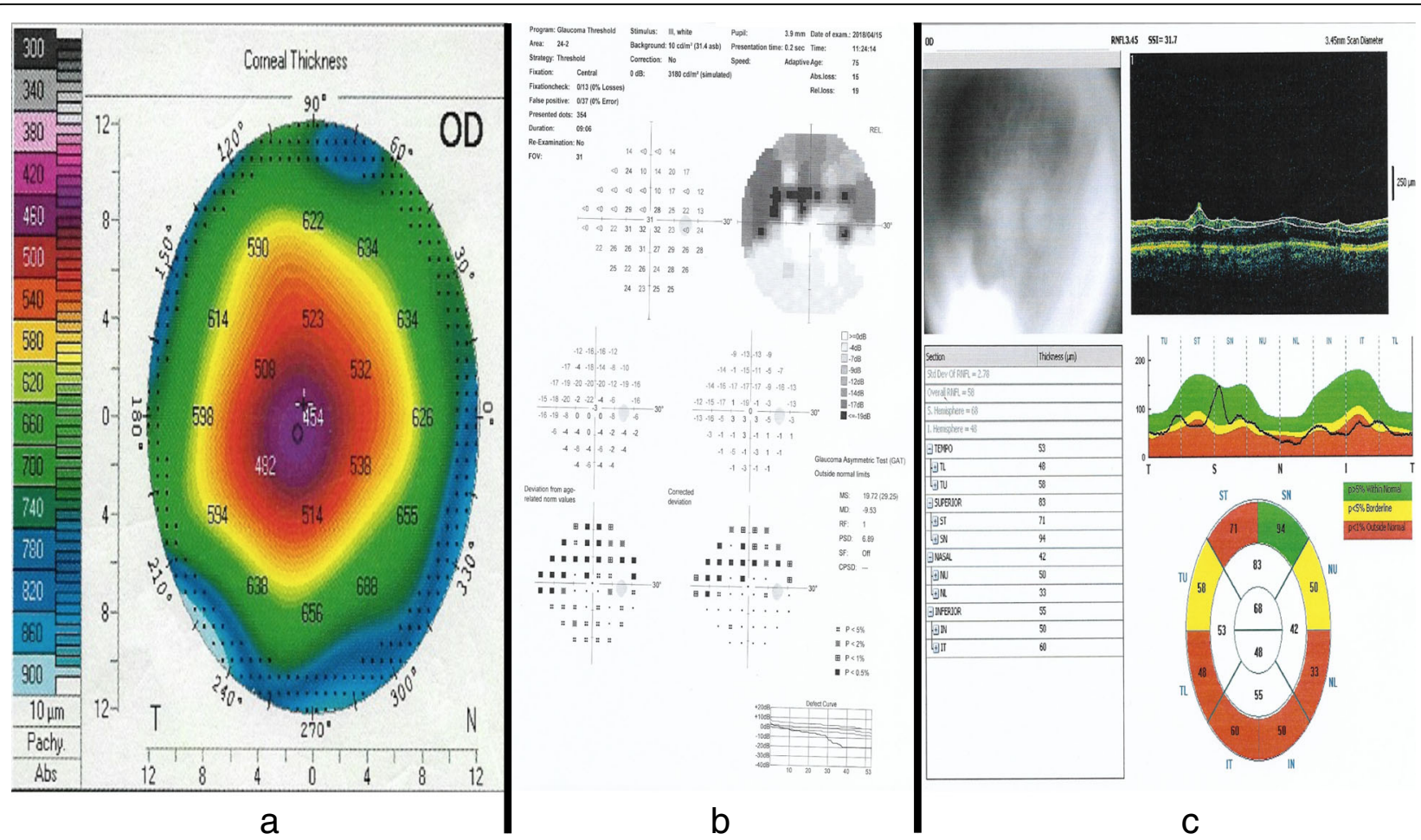

Fig. 1 Imaging in a patient with advanced chronic angle closure glaucoma. a Pentacam printout of right eye corneal thickness map showing a central corneal thickness (CCT)of $454 \mu \mathrm{m}$, which is significantly thinner than average CCT of 530-550 $\mu \mathrm{m}$. b Threshold 24-2 Visual Field test of the right eye demonstrating a superior arcuate scotoma splitting fixation. c Ocular coherence tomography of the retinal nerve fiber layer thickness (OCT RNFL) of the right eye showing significant thinning (Overall RNFL $=58$ ). 
advanced visual field defects, and thinner RNFL. We also found that patients with PACG had the lowest CCT compared to POAG, PXFG, and controls. An Iranian study, that included both POAG and PACG patients, found that POAG patients have lower CCT compared to their PACG counterparts [18]. Regarding PXFG, a Caucasian study found a significantly lower CCT in PXFG patients compared to POAG patients [19], this emphasizes the profound difference in CCT in different ethnic groups and the importance of $\mathrm{CCT}$ as a risk factor for progression of glaucoma.

The strong relation between CCT and ethnicity is well-studied. A large study that was done on a Chinese population to study the relation between CCT and around 20 variables found that ethnicity is one of the important determinants of CCT [20]. A worldwide meta-analysis found that the average CCT for normal eyes derived from various racial groups, regardless of the device used, was $544 \mu \mathrm{m}$ [21], a value that is thinner than that for the Jordanian population we studied $(550 \mu \mathrm{m})$. Comparing CCT values of our Jordanian population with Caucasian ethnicity, the most represented ethnicity in ethnicity-comparing studies [22], Caucasian ethnicity mean CCT was similar to Jordanian's $(550 \pm 30.5 \mu \mathrm{m})$ [23]. On the other hand, African ethnicity has a lower CCT than that for Jordanians (514.77 \pm 31.86$)$ [23]. It should be noted that the technique used to measure CCT can affect the results [24], and although the results from commonly used devices mostly correlate, the direct comparison between them might not be accurate [22], so that we will only include studies that used similar techniques for direct value comparison purposes. Regarding CCT difference between different glaucoma types, only PACG eyes have thinner corneas compared to POAG, PXFG, and control eyes, This comes in accordance with what a recent study suggested, where no difference in corneal thickness between POAG, PXFG, and control eyes was found [25].

Other reports generally have results that are comparable to ours, except for a study by Tolesa and Gessesse that was done on Ethiopian patients [26], and found thicker CCT in patients with POAG $(520 \pm 38.95)$ compared to PXFG patients $(507 \pm 35)$.

Several previous studies have reported the association between larger CDR and glaucomatous progression [27]. Here, we found that patients with larger CDR have worse mean deviation (MD) compared to those with smaller ratios. In Ocular Hypertension Treatment Study [28], they found that cup to disc ratio is also a predictor of the development of POAG in normal subjects. A recent study found that for every 0.1-unit increase in the CDR, there is an increase in the decay rate of visual field by $23 \%$ [29]. We also found that the largest CDR is found in PACG patients, followed by POAG, and finally PXFG.
Similarly, we found that RNFL thickness measured by Optical Coherence Tomography (OCT) is also a good indicator for the severity of visual field loss represented by the mean deviation (MD). In a 12-year study, the risk estimate for moderate and severe visual field defects found to suggest a seven to eight times greater likelihood for future field loss for eyes with initial atrophy [30]. Where previous reports found an association between IOP and MD [30], we think that this effect is indirect through affecting the CDR, as the relation between IOP and MD did not reach significance when we corrected for CDR, probably because these were treated IOP readings.

Several limitations of this study exist. We advice future studies to include only one eye for each included subject (independence of observation) to overcome correlated observation issue as explained in a previous study, although it can be corrected using certain statistical tests [31]. Moreover, a longitudinal approach to follow Middle Eastern patients would provide a better insight on the outcome of different CCT. Although this is a multicenter study, future studies from other Arabian countries should consider including larger sample size to confirm the generalizability of our results.

\section{Conclusion}

We found that PACG patients have the thinnest CCT compared to other types of glaucoma, namely POAG and PXFG. We also demonstrated that glaucomatous eyes with thinner corneas will probably have more advanced glaucomatous optic neuropathy. This emphasizes the importance of making CCT measurement a part of standard care in all glaucoma patients since it can predict patients with worse prognosis, and therefore can indicate the need for more aggressive management and earlier diagnosis. Finally, we showed that cup to disc ratio, retinal nerve fiber layer thickness and mean deviation on visual field testing usually are in concordance regarding the severity of glaucoma.

\section{Abbreviations \\ CCT: Central corneal thickness; CDR: Cup-to-disc ratio; IOP: Intraocular pressure; MD: Visual field mean deviation; OCT: Ocular coherence tomography; PACG: Primary angle closure glaucoma; POAG: Primary open angle glaucoma; PSD: Pattern standard deviation; PXFG: Pseudoexfoliative glaucoma; RNFL: Retinal nerve fiber layer}

Acknowledgements

We would like to thank Dr.Doukhi Hunaiti, The University of Jordan for his professional statistical consultation during our statistical analysis review.

\section{Funding}

None

\section{Availability of data and materials}

The datasets during and/or analyzed during the current study are available from the corresponding author on reasonable request. 


\section{Authors' contributions}

Conception and design of the study: SM, SAA. Acquisition of data: FA, MH. Analysis and interpretation of data: SM, SAA. Drafting of the manuscript: SM, SAA. Critical revision of the manuscript: SM, SAA, MH. All authors read and approved the final version to be published.

\section{Ethics approval and consent to participate}

An approval for the performance of the study was obtained from the Ethics Committee of the University of Jordan. All patients were informed previously about the study and signed an informed consent in accordance with the tenets of the Helsinki Declaration.

\section{Consent for publication}

Not applicable.

\section{Competing interests}

The authors declare that they have no competing interests

\section{Publisher's Note}

Springer Nature remains neutral with regard to jurisdictional claims in published maps and institutional affiliations.

\section{Author details}

'Ophthalmology, Glaucoma and Anterior Segment Surgeon, University of Jordan Hospital, The University of Jordan, Amman, Jordan. ${ }^{2}$ School of Medicine, The University of Jordan, Amman 11942, Jordan. ${ }^{3}$ Department of Ophthalmology, University of Jordan Hospital, The University of Jordan, Amman 11942, Jordan.

\section{Received: 25 October 2017 Accepted: 16 October 2018}

\section{Published online: 29 October 2018}

\section{References}

1. Quigley HA, Broman AT. The number of people with glaucoma worldwide in 2010 and 2020. Br J Ophthalmol. 2006;90(3):262-7.

2. Jonasson F, Damji KF, Arnarsson A, Sverrisson T, Wang L, Sasaki H, Sasaki K. Prevalence of open-angle glaucoma in Iceland: Reykjavik eye study. Eye. 2003:17(6):747

3. Palko JR, Qi O, Sheybani A. Corneal alterations associated with pseudoexfoliation syndrome and glaucoma: a literature review. J Ophthal Vision Res. 2017;12(3):312.

4. Dimasi DP, Burdon KP, Craig JE. The genetics of central corneal thickness. $\mathrm{Br}$ J Ophthalmol. 2010;94(8):971-6.

5. Goldmann HA. The glaucoma problem. InTrans. 2nd. Glaucoma Conf; 1957. p. 137-65.

6. Hansen FK, Ehlers N. Elevated tonometer readings caused by a thick cornea. Acta Ophthalmol. 1971:49(5):775-8

7. Doughty MJ, Laiquzzaman M, Müller A, Oblak E, Button NF. Central corneal thickness in European (white) individuals, especially children and the elderly, and assessment of its possible importance in clinical measures of intraocular pressure. Ophthalmic Physiol Opt. 2002;22(6):491-504.

8. La Rosa FA, Gross RL, Orengo-Nania S. Central corneal thickness of Caucasians and African Americans in glaucomatous and nonglaucomatous populations. Arch Ophthalmol. 2001;119(1):23-7.

9. Aghaian E, Choe JE, Lin S, Stamper RL. Central corneal thickness of Caucasians, Chinese, Hispanics, Filipinos, African Americans, and Japanese in a glaucoma clinic. Ophthalmology. 2004;111(12):2211-9.

10. Wang SY, Melles R, Lin SC. The impact of central corneal thickness on the risk for glaucoma in a large multiethnic population. J Glaucoma. 2014;23(9):606

11. Bechmann M, Thiel MJ, Roesen B, Ullrich S, Ulbig MW, Ludwig K. Central corneal thickness determined with optical coherence tomography in various types of glaucoma. Br J Ophthalmol. 2000;84(11):1233-7.

12. Baarah BT, Shatnawi RA, Khatatbeh AE. Causes of permanent severe visual impairment and blindness among Jordanian population. Middle East Afr $J$ Ophthal. 2018;25(1):25

13. Al-Zubi K, Sarayrah F, Al-Awaishah M. Glaucoma awareness and knowledge among Jordanian people. Global J Health Sci. 2017:9(8):40.

14. Rudnicka AR, Mt-Isa S, Owen CG, Cook DG, Ashby D. Variations in primary open-angle glaucoma prevalence by age, gender, and race: a Bayesian meta-analysis. Invest Ophthalmol Vis Sci. 2006;47(10):4254-61.
15. Cedrone C, Mancino R, Cerulli A, Cesareo M, Nucci C. Epidemiology of primary glaucoma: prevalence, incidence, and blinding effects. Prog Brain Res. 2008;173:3-14.

16. Lin W, Aoyama Y, Kawase K, Yamamoto T. Relationship between central corneal thickness and visual field defect in open-angle glaucoma. Jpn J Ophthalmol. 2009:53(5):477-81.

17. Jonas JB. Clinical implications of peripapillary atrophy in glaucoma. Curr Opin Ophthalmol. 2005;16(2):84-8.

18. Moghimi S, Zandvakil N, Vahedian Z, Mohammadi M, Fakhraie G, Coleman $\mathrm{AL}$, Lin SC. Acute angle closure: qualitative and quantitative evaluation of the anterior segment using anterior segment optical coherence tomography. Clin Exp Ophthalmol. 2014;42(7):615-22.

19. Kitsos G, Gartzios C, Asproudis I, Bagli E. Central corneal thickness in subjects with glaucoma and in normal individuals (with or without pseudoexfoliation syndrome). Clin Ophthal (Auckland, NZ). 2009:3:537.

20. Pan CW, Li J, Zhong H, Shen W, Niu Z, Yuan Y, Chen Q. Ethnic variations in central corneal thickness in a rural population in China: the Yunnan minority eye studies. PLoS One. 2015;10(8):e0135913.

21. Doughty MJ, Zaman ML. Human corneal thickness and its impact on intraocular pressure measures: a review and meta-analysis approach. Surv Ophthalmol. 2000;44(5):367-408.

22. Belovay GW, Goldberg I. The thick and thin of the central corneal thickness in glaucoma. Eye. Feb. 2018;15:1.

23. Baboolal SO, Smit DP. South African Eye Study (SAES): ethnic differences in central corneal thickness and intraocular pressure. Eye. 2018;32:749-56.

24. Wong AC, Wong CC, Yuen NS, Hui SP. Correlational study of central corneal thickness measurements on Hong Kong Chinese using optical coherence tomography, Orbscan and ultrasound pachymetry. Eye. 2002;16(6):715.

25. Ayala MJ, Pérez-Santonja JJ, Artola A, Claramonte P, Alió JL. Laser in situ keratomileusis to correct residual myopia after cataract surgery. J Refract Surg. 2001;17(1):12-6.

26. Tolesa K, Gessesse GW. Central corneal thickness in newly diagnosed glaucoma patients in south West Ethiopia: a cross-sectional study. BMC Ophthalmol. 2016;16(1):152.

27. Nicolela MT, Drance SM, Broadway DC, Chauhan BC, McCormick TA, LeBlanc RP. Agreement among clinicians in the recognition of patterns of optic disk damage in glaucoma. Am J Ophthalmol. 2001;132(6):836-44.

28. Gordon MO, Beiser JA, Brandt JD, Heuer DK, Higginbotham EJ, Johnson CA, Keltner JL, Miller JP, Parrish RK, Wilson MR, Kass MA. The ocular hypertension treatment study: baseline factors that predict the onset of primary openangle glaucoma. Arch Ophthalmol. 2002;120(6):714-20.

29. Lee JM, Cirineo N, Ramanathan M, Nouri-Mahdavi K, Morales E, Coleman AL, Caprioli J. Performance of the visual field index in glaucoma patients with moderately advanced visual field loss. Am J Ophthalmol. 2014;157(1):39-43.

30. Quigley HA, Enger C, Katz J, Sommer A, Scott R, Gilbert D. Risk factors for the development of glaucomatous visual field loss in ocular hypertension. Arch Ophthalmol. 1994;112(5):644-9.

31. Sainani $K$. The importance of accounting for correlated observations. PM\&R 2010;2(9):858-61.

Ready to submit your research? Choose BMC and benefit from:

- fast, convenient online submission

- thorough peer review by experienced researchers in your field

- rapid publication on acceptance

- support for research data, including large and complex data types

- gold Open Access which fosters wider collaboration and increased citations

- maximum visibility for your research: over $100 \mathrm{M}$ website views per year

At $\mathrm{BMC}$, research is always in progress.

Learn more biomedcentral.com/submission 